\section{AROTULAGEM NUTRICIONAL COMO FERRAMENTA PARA A PROMOÇÃO DO AUTOCUIDADO EM DIABÉTICOS}

\author{
Nutrition labeling as a tool to promote self-care in diabetic \\ patients
}

\section{La rotulación nutricional como herramienta para la promoción del autocuidado de diabéticos}

\section{RESUMO}

Objetivo: Descrever o processo de elaboração e execução de oficinas sobre rotulagem nutricional, contextualizada às necessidades de pacientes diabéticos. Síntese dos dados: Realizou-se um trabalho com integrantes de um grupo de diabéticos da cidade de Currais Novos-RN. Inicialmente, fez-se uma avaliação da legislação vigente sobre rotulagem, buscando identificar pontos de impacto para o paciente diabético. Então, foram selecionados os seguintes temas: diet, light e zero; alimento integral; nomenclaturas e sinônimos para designar açúcar; ordem dos ingredientes; instruções de uso e porção/medida caseira. A seguir, elaborou-se um material de apoio visual para melhor assimilação das informações e idealizaram-se atividades de grupo, buscando oferecer vivências que permitissem trabalhar de forma mais ágil e prática alguns pontos, favorecendo a compreensão das informações. Os participantes aderiram a essas atividades, mostrando-se participativos, além de conseguirem vislumbrar a aplicação, em seu cotidiano, dos conhecimentos adquiridos. Conclusão: As oficinas contribuíram para o aprofundamento de conhecimentos e a aquisição de habilidades para lidar com o diabetes, incentivando o protagonismo dos pacientes em relação ao cuidado dietético. Diante da boa aceitação dessa iniciativa e do potencial que o tipo de informação trabalhada tem para melhorar a qualidade de vida de quem vive com diabetes, espera-se que atividades semelhantes sejam inseridas com maior frequência no repertório dos serviços de cuidado à saúde.

Descritores: Rotulagem de Alimentos; Educação Nutricional; Diabetes.

\section{ABSTRACT}

Objective: To describe the process of developing and implementing a workshop on nutrition labeling tailored to the needs of diabetic patients. Data synthesis: The study was conducted with members of a diabetes group in Currais Novos/RN. Initially, a review of the existing legislation on labeling was made in order to identify impact points for the diabetic patient. Then, the following themes were selected: diet, light and zero; wholesome food; nomenclature and synonyms to designate sugar; order of ingredients; instructions for use and serving/portion size. After that, a visual support material was elaborated for a better assimilation of information and group activities were devised seeking to offer experiences that would allow to work on some issues in a more agile and practical way, favoring the understanding of information. The individuals actively participated in the activities and also got a glimpse of how to apply the acquired knowledge to their daily lives. Conclusion: The workshops contributed to the deepening of knowledge and the acquisition of skills to handle diabetes, encouraging the protagonism of patients in relation to dietary care. Given the good acceptance of this initiative and the power that such type of information has to improve the quality of life of diabetic patients, it is expected that similar activities are included more often in the repertoire of health care services.

Descriptors: Food labeling; Nutrition Education; Diabetes.
Descrição ou avaliação de experiências, métodos, técnicas, procedimentos e instrumentais

Anna Cecília Queiroz de Medeiros $^{(1)}$

Amanda Laís Pereira de Lima ${ }^{(1)}$ Júlia da Nóbrega Silva ${ }^{(1)}$ Luana Joselyn Silva do Nascimento $^{(1)}$

1) Faculdade de Ciências da Saúde do Trairi - FACISA - Universidade Federal do Rio Grande do Norte - UFRN - Santa Cruz $(\mathrm{RN})$ - Brasil
Recebido em: 01/03/2014 Revisado em: 25/05/2014 Aceito em: 03/09/2014 


\section{RESUMEN}

Objetivo: Describir el proceso de elaboración y ejecución de talleres de rotulación nutricional en el contexto de las necesidades de pacientes diabéticos. Síntesis de los datos: Se realizó un trabajo con los integrantes de un grupo de diabéticos de la ciudad de Currais Novos-RN. A principio se hizo una evaluación de la legislación en vigor sobre rotulación intentando identificar los puntos de impacto para el paciente diabético. Fueron seleccionados los siguientes temas: diet, light y zero; el alimento integral; las nomenclaturas y sinónimos para designar el azúcar; el orden de los ingredientes; las instrucciones de uso y la porción/medida casera. En seguida, se elaboró un material de apoyo visual para una mejor asimilación de las informaciones y se idealizo las actividades de grupo intentado ofrecer vivencias que permitieran trabajar de manera más ágil y práctica algunos puntos que facilitara la compresión de las informaciones. Los participantes adhirieron a las actividades con participación, además de lograren vislumbrar la aplicabilidad de los conocimientos adquiridos en su cotidiano. Conclusión: Los talleres contribuyeron para la profundización de los conocimientos y la adquisición de las habilidades para convivir con la diabetes, incentivando el protagonismo de los pacientes sobre el cuidado dietético. Frente la buena aceptación de la iniciativa y del potencial de la información trabajada para mejorar la calidad de vida de uno que vive con diabetes se espera que actividades semejantes sean insertadas con más frecuencia en los servicios de cuidados a la salud.

Descriptores: Etiquetado de alimentos; Educación Alimentaria y Nutricional; Diabetes Mellitus.

\section{INTRODUÇÃO}

Em 2007, apenas quatro doenças crônicas, dentre as quais está o diabetes, foram responsáveis por $58 \%$ das mortes no Brasil ${ }^{(1)}$. Parte dessa morbimortalidade é atribuída às modificações no padrão alimentar da população, principalmente pelo aumento no consumo de calorias, gordura animal e alimentos industrializados, além de diminuição na ingestão de fibras, frutas e verduras ${ }^{(2,3)}$.

Por isso, o consumo alimentar pode ser considerado um determinante da saúde, cujo caráter positivo ou negativo depende das escolhas feitas no cotidiano, quando da compra, aquisição e consumo de alimentos ${ }^{(4)}$. Para melhor subsidiar essas práticas, por parte da população, é de fundamental importância a execução de ações, políticas e atividades que auxiliem a população na adoção de uma dieta mais saudável ${ }^{(5,6)}$.

Uma iniciativa importante é a padronização da rotulagem nutricional, definida como toda descrição destinada a informar o consumidor sobre as propriedades nutricionais de um alimento, compreendendo a declaração de valor energéticos e os principais nutrientes ${ }^{(7)}$.

No Brasil, a rotulagem nutricional é regulamentada pelas Resoluções de Diretoria Colegiada (RDCs) da Agência Nacional de Vigilância Sanitária (ANVISA) ${ }^{(8-}$ 10). Essas informações fornecidas por meio da rotulagem devem ser claras e com especificação correta de quantidade, composição e qualidade dos produtos aos quais se referem, bem como sobre propriedades e/ou alegações de saúde inerentes a seu consumo ${ }^{(7,11)}$.

A veracidade das informações apresentadas pelo rótulo nutricional em alimentos deve ser garantida, para que essa ferramenta cumpra o objetivo de auxiliar o consumidor em suas escolhas e os profissionais de saúde na orientação para a composição da dieta ${ }^{(7,12)}$.

Nessa perspectiva, as informações contidas nos rótulos permitem ao consumidor o acesso a parâmetros nutricionais e indicativos da qualidade e segurança do alimento, constituindo-se uma ferramenta de grande valor para a construção da responsabilidade pessoal em relação ao consumo de alimentos ${ }^{(13)}$.

Entretanto, para que possam atuar como um instrumento de educação nutricional e alimentar, é necessário que essas informações sejam compreendidas por todos aqueles que as utilizam $^{(5,6)}$. No entanto, nem sempre as pessoas costumam ler a informação nutricional presente nas embalagens dos alimentos que consomem ${ }^{(7)}$, e quando o fazem, não compreendem adequadamente o seu significado ${ }^{(5,14,15)}$.

Particularmente no caso de pacientes diabéticos, que possuem condições metabólicas e fisiológicas diferenciadas, cujo controle e manejo requerem a adoção de uma dieta específica, esse conhecimento pode ser de grande valia no processo de autocuidado ${ }^{(16,17)}$.

É por isso que a educação nutricional, no controle do diabetes, deve estar focada no desenvolvimento da capacidade dos indivíduos de identificar possibilidades de melhoria em sua dieta, refletindo sobre suas motivações e contribuindo para a construção de uma consciência crítica $^{(13,16,18)}$.

Um padrão alimentar adequado é fundamental para o controle glicêmico no diabetes, bem como para a prevenção de complicações e sequelas da doença, sendo a realização de atividades educativas sobre o tema um aspecto muito relevante no tratamento ${ }^{(17,19)}$. Mas para o sucesso da educação desses pacientes, é imprescindível considerar os aspectos motivacionais para o autocuidado, a participação da família e o estabelecimento de vínculos efetivos com a equipe multiprofissional. O desafio está em sempre buscar um momento e um espaço para educar ${ }^{(20)}$. 
Nesse sentido, as atuais "Diretrizes para o cuidado das pessoas com doenças crônicas nas redes de atenção à saúde e nas linhas de cuidado prioritárias" preconizam as atividades em grupo como um momento ímpar para a educação em saúde, permitindo o intercâmbio de informações e experiências entre usuários e equipes de saúde, além de fomentar práticas de autocuidado e mudança de atitude ${ }^{(21)}$. Outrossim, trata-se de um recurso que pode auxiliar na melhor conscientização da importância da responsabilização pelo tratamento por parte do paciente como agente atuante, e não apenas como mero recebedor de prescrições ${ }^{(13,18,21)}$.

Considerando, então, a importância da rotulagem nutricional para fornecer informações que ajudem na avaliação do alimento e, consequentemente, na escolha do repertório alimentar, evidencia-se a necessidade de educar os consumidores para entender melhor esses dados.

Assim, este trabalho teve por objetivo descrever o processo de elaboração e execução de oficinas sobre rotulagem nutricional, contextualizada às necessidades de pacientes diabéticos.

\section{SÍNTESE DE DADOS}

Tratou-se da descrição de experiência realizada com integrantes do grupo de diabéticos Renascer, constituído de 832 participantes, vinculados ao Hospital Regional Dr. Mariano Coelho, localizado na cidade de Currais Novos, interior do Rio Grande do Norte. Diante do grande número de integrantes, dividiram-se os participantes em grupos de 30 pessoas e a oficina de rotulagem foi ministrada várias vezes.

Para a elaboração das oficinas, primeiramente, fez-se uma análise das resoluções da ANVISA sobre rotulagem ${ }^{(8-10)}$, visando selecionar os temas que possuíssem maior relevância e impacto para o dia a dia dos pacientes com diabetes. Os pontos escolhidos foram: a definição e a diferença entre os termos diet, light e zero; "o que é um alimento integral?"; as múltiplas nomenclaturas que o açúcar pode apresentar nos rótulos de alimentos; o significado da ordem dos ingredientes que constituem os produtos; além das instruções de uso e porção/medida caseira que cada alimento possui.

A seguir, o grupo de tutores realizou encontros para aprofundar os conhecimentos sobre os temas de rotulagem de alimentos, diabetes e educação nutricional, de modo a melhor subsidiar a elaboração do material e definir as estratégias educativas para a oficina.

As informações foram organizadas em slides, sendo projetados em multimídia, priorizando a utilização de figuras e desenhos, para melhor fluidez da exposição e compreensão dos conteúdos pelos participantes de menor escolaridade. Também se definiu, enquanto elemento norteador na elaboração das oficinas, a necessidade de incluir vivências e/ou experiências práticas, visando uma participação mais ativa e maior envolvimento dos diabéticos no processo de construção do conhecimento.

Realizaram-se novos encontros pelo grupo de tutores para discutir e avaliar os materiais elaborados, os quais foram repetidamente ajustados até que se considerasse o resultado satisfatório do ponto de vista nutricional e pedagógico. Dentre os critérios utilizados para definir a adequabilidade, do ponto de vista pedagógico, estavam: maior proporção de informações em forma de figuras e fluxogramas; boa qualidade gráfica; quantidade de informação por slide; clareza nos conceitos; exequibilidade das dinâmicas propostas (tempo necessário, materiais e tipo de participação envolvida); e utilização de linguagem correta, embora coloquial.

Outro aspecto considerou que alimentos, receitas e produtos utilizados em exemplos, figuras e atividades fizessem parte do repertório disponível na região e fossem economicamente acessíveis e/ou de fácil preparo, uma vez que os membros do grupo possuíam, em sua maioria, idade superior a 50 anos e baixo poder aquisitivo.

Para exemplificar os conceitos de diet e light, a porção/ medida caseira e sinonímias do açúcar, utilizou-se como ideia condutora as informações nutricionais presentes no rótulo de um biscoito diet de fácil aquisição no mercado local, bem como a sua apresentação entre os participantes da oficina. À medida que a discussão ia avançando, outros alimentos também apareciam em cena, como os refrigerantes, que também eram de marcas conhecidas, sendo empregados para melhor elucidar o que era um produto zero.

No ponto referente às diferenças entre diet e light, basearam-se os conceitos básicos na resolução específica para alimentos com fins especiais. A questão da diminuição ou restrição do ingrediente nos alimentos foi a base central da apresentação, pontuada pela grande participação dos presentes, que consumiam os alimentos utilizados e conseguiam transcender as informações para seu cotidiano, dirimindo dúvidas e reconstruindo conceitos e valores já estabelecidos.

Um momento de grande inquietação entre os participantes era quando se falava sobre as diversas nomenclaturas do açúcar apresentadas nos rótulos dos produtos (açúcar mascavo, açúcar simples, xarope de glicose, glucose de milho, glicose e glaçúcar, por exemplo). A maioria dos diabéticos presentes não conseguia relacionar essas palavras a açúcar, ou achava, por exemplo, que sendo açúcar mascavo não haveria problema. Esse tópico teve a finalidade de alertá-los e capacitá-los para a identificação 
do açúcar nos alimentos e para a análise da adequabilidade de alimentos light para o seu consumo.

De fato, muitos participantes manifestaram dúvidas sobre as terminologias diet e light, com a não definição correta desses termos ou até a troca entre ambos. Esse tipo de dúvida pode tanto limitar ainda mais o repertório alimentar do diabético, que pode se sentir inseguro sobre consumir ou não algum alimento, como também pode levar a escolhas inadequadas, ricas em açúcar, contribuindo para a piora do controle glicêmico.

O termo light é utilizado em alimentos que obtenham $25 \%$ de redução em qualquer nutriente comparado ao original. Da mesma forma, pode ser utilizado o termo "baixo" ou "pobre". Diferente dos alimentos diet, em que há restrição total de açúcares. Entretanto, os alimentos light não necessariamente têm redução no conteúdo calórico de ou açúcares, podendo haver até mesmo redução no conteúdo de proteínas ou fibras e ser designado no rótulo como um produto light ${ }^{(8)}$.

Uma situação frequentemente encontrada e discutida na oficina foi que, em alguns casos, os alimentos light não têm açúcar em sua composição, sendo adequados para o diabético. No entanto, a menos que realmente compreenda o significado das terminologias diet e light, e seja capaz de identificar a presença de açúcar na lista de ingredientes do alimento, o paciente diabético não terá como definir se um alimento light é ou não adequado para seu consumo.

Para explorar o conceito do que seria o alimento integral, escolheram-se os exemplos do leite e do arroz, que aparecem em quase todas as recomendações para uma dieta saudável do diabético ${ }^{(22)}$. Primeiramente, foi sendo construída a ideia do que seria a palavra "integral", por si só, de modo a desatrelá-la da ideia de saudável. Nesse momento, discutiu-se o caso do leite, que poderia ser encontrado na forma integral, semidesnatadado e desnatado. Apresentaram-se imagens dos produtos, as diferenças entre as composições e se discutiu as indicações de uso que eles haviam recebido de seus profissionais de saúde. Em seguida, foram mostradas as diferenças entre arroz integral, polido, "da terra" e parboilizado, enfocando na composição nutricional, particularmente em relação ao teor de fibras e às respostas glicêmicas que eles provocam com sua ingestão. O objetivo final era que, para além de simplesmente aceitar o nome "integral", expresso no rótulo, se buscasse entender o porquê de o alimento ser integral e se a presença desses componentes seria adequada ou não para o diabético.

Trabalhou-se a noção de porção e medida caseira a partir do biscoito diet, cujas embalagens, lacradas, circulavam entre os presentes. Solicitou-se que alguns abrissem os pacotes e separassem a quantidade que normalmente consumiriam ou que identificavam como sendo uma porção. A seguir, lia-se no pacote qual quantidade correspondia à medida de porção, segundo a legislação vigente, e a quantidade era então separada. A partir desse momento, todos eram convidados a comparar os montantes, debater possíveis diferenças e repensar o conceito de porção, a partir das indicações disponíveis nos rótulos dos alimentos. Também era discutido o fato de que as informações nutricionais contidas no rótulo, como carboidratos, gorduras, proteínas, sódio, são referentes a uma porção do produto que nem sempre equivale a todo o conteúdo da embalagem.

Quando se abordou a questão das instruções de uso, os alimentos adotados para nortear as atividades foram o adoçante e o creme vegetal, também de marcas populares. Imagens da embalagem de creme vegetal e de partes ampliadas foram utilizadas para demonstrar que, muitas vezes, não se utiliza os alimentos de maneira correta, o que pode trazer prejuízos à saúde. No caso específico do creme vegetal, mostrou-se a recomendação de armazenamento sob refrigeração e de não levar o produto ao fogo.

Essas recomendações foram feitas porque, quando mantido em temperatura ambiente, o creme vegetal, rico em gorduras insaturadas, fica muito propenso aos processos oxidativos. Essa oxidação pode converter a gordura insaturada em gordura saturada, cujo excesso está relacionado ao aumento do risco para comorbidades do diabetes. Devido ao seu alto teor de água, o creme vegetal também não deve ser submetido ao aquecimento, pois faz com que aconteçam mudanças em sua textura e também pode alterar o perfil da gordura ${ }^{(23)}$. Entretanto, a maioria dos presentes nunca havia percebido que esses avisos estavam impressos na embalagem.

No caso da recomendação de uso do adoçante, foi proposta uma atividade na qual alguns participantes eram convidados a pegar um frasco de adoçante, que estava cheio de líquido colorido, e colocar em um pires a quantidade que normalmente utilizaria para uma xícara de café, da maneira como faria em casa. Muitas vezes, os pacientes simplesmente "chiringavam" o produto, apertando o frasco e contando as apertadas. Foi lida então a recomendação de utilização do produto e realizado o porcionamento conforme descrito. Em seguida, os pires foram colocados lado a lado e todos foram convidados a observar e comparar os conteúdos. Percebeu-se que muitos pacientes desconheciam a recomendação de uso para adoçantes, o que gerou um debate sobre a importância de ler e seguir as instruções contidas nos rótulos.

Embora os adoçantes sejam rotineiramente utilizados como substitutos do açúcar na alimentação diária, devem ser tomadas certas precauções quanto ao seu consumo, como limitar a quantidade de acordo com as recomendações de ingestão diária aceitável (IDA) vigentes ${ }^{(24,25)}$. 
Para trabalhar a importância da ordem dos ingredientes no rótulo, realizou-se uma breve explanação sobre o assunto, seguida de uma estratégia para trabalho em grupo. Divididos em duas equipes, os participantes deveriam organizar os ingredientes de uma receita, de acordo com as normas de rotulagem. A receita de cada grupo era disponibilizada em uma projeção, acessível a todos, com as quantidades em gramas de cada ingrediente. Cada grupo recebia, então, fichas grandes de cartolina, com os nomes dos ingredientes escritos, as quais deveriam ser afixadas na parede, organizadas como se estivessem no rótulo do produto a ser confeccionado com a receita. Houve participação efusiva dos grupos nessa atividade.

A ordem dos ingredientes apresentada nas embalagens dos alimentos indica, no sentido decrescente, a quantidade de cada ingrediente que compõe o produto, ou seja, os primeiro da lista é o que tem maior quantidade em relação aos demais ${ }^{(26)}$.

Assim, é importante observar em qual posição o açúcar, ou outro nome que ele receba, está em relação ao total de ingredientes listados. O objetivo dessa atividade era despertar a atenção dos participantes para essa informação, a fim de que incorporassem tal prática rotineiramente, no momento de aquisição e consumo de alimentos industrializados.

Em decorrência da realização das oficinas de rotulagem de alimentos, percebeu-se a necessidade de se realizar, continuamente, ações educativas com o público diabético. Apesar de a maioria das informações transmitidas ser bastante simples e amplamente divulgada pela mídia, houve assuntos desconhecidos e outros a respeito dos quais não havia clareza sobre sua adequada operacionalização, apesar de serem conhecidos.

Por exemplo, no caso do adoçante, muitos participantes já sabiam quantas gotas de adoçante colocar, mas não sabiam as consequências da superdosagem e, quando foi explicado, todos entenderam a importância de seguir o rótulo. Por outro lado, nenhum dos participantes, em nenhuma das sessões da oficina, tinha conhecimento sobre o significado da ordem dos ingredientes ou sobre sua relação com a constituição e/ ou formulação dos produtos.

Percebe-se então que as atividades de educação alimentar e nutricional devem ser planejadas e executadas de modo que os conhecimentos trabalhados possam ser percebidos como algo próximo, tangível à realidade da comunidade. Ou seja, que haja uma real apropriação das informações.

A literatura sugere ${ }^{(18,27)}$ que uma das maneiras de conseguir abranger esse aspecto seria por meio de atividades e estratégias norteadas pelo conceito de empowerment (empoderamento). A técnica de empoderamento surgiu por volta das décadas de 1980 e 1990, simbolizando uma ação coletiva desenvolvida pelos indivíduos quando participam de espaços privilegiados de decisões, de consciência social dos direitos sociais. Essa consciência ultrapassa a tomada de iniciativa individual de conhecimento e superação de uma realidade, possibilitando a aquisição da emancipação individual e também da consciência coletiva necessária para a superação da dependência social e dominação política ${ }^{(28)}$.

Assim, um agente externo, um profissional de saúde, empodera indivíduos e comunidades oferecendo informações e treinamento para que possam promover mudanças em suas vidas, de modo que a intervenção transforme indivíduos em senhores de sua vida cotidiana, adotando práticas alimentares ou atividades físicas prédefinidas como mais saudáveis, desconsiderando as desigualdades sociais, de gênero e raciais que atravessam as práticas e cotidianos dos participantes - e dos profissionais de saúde - nas ações de promoção de práticas alimentares saudáveis ${ }^{(20,29)}$.

No contexto particular do diabetes, a adesão a uma dieta adequada permite uma diminuição no risco do surgimento de comorbidades, além de proporcionar maior qualidade de vida ao paciente ${ }^{(17,21)}$. Diante do processo dinâmico que é a escolha alimentar, das inúmeras possibilidades diárias com as quais o diabético precisa lidar, atividades de educação alimentar e nutricional podem auxiliar no desenvolvimento de capacidades para melhor enfrentar estas situações.

A oficina de rotulagem de alimentos foi construída na perspectiva do empoderamento, uma vez que as informações compartilhadas possuem grande potencial para influenciar nas escolhas alimentares dos participantes e em sua forma de lidar com a doença, fomentando habilidades e competências possíveis de serem incorporadas ao cotidiano dos participantes.

\section{CONCLUSÃO}

As oficinas contribuíram para o aprofundamento de conhecimentos e a aquisição de habilidades para lidar com o diabetes, incentivando o protagonismo dos pacientes em relação ao cuidado dietético. Diante da boa aceitação dessa iniciativa e do potencial que o tipo de informação trabalhada tem para melhorar a qualidade de vida de quem vive com diabetes, espera-se que atividades semelhantes sejam inseridas com maior frequência no repertório dos serviços de cuidado à saúde.

\section{AGRADECIMENTOS}

À Fundação Padre João Maria do Hospital Dr. Mariano Coelho - Município de Currais Novos-RN e aos professores Ênio Walker de Azevedo Cacho e Edvaldo Vasconcelos de Carvalho Filho. 


\section{REFERÊNCIAS}

1. Ministério da Saúde (BR), Secretaria de Vigilância em Saúde, Departamento de Análise da Situação de Saúde. Plano de ações estratégicas para o enfrentamento das doenças crônicas não transmissíveis (DCNT) no Brasil/2011-2022. Brasília: Ministério da Saúde; 2011.

2. Gimeno SGA, Mondini L, Moraes SA, Freitas ICM. Padrões de consumo de alimentos e fatores associados em adultos de Ribeirão Preto, São Paulo, Brasil: Projeto OBEDIARP. Cad Saúde Pública. 2011;27(3):533-45.

3. Lenz A, Olinto MTA, Dias-da-Costa JS, Alves AL, Balbinotti M, Pattussi MP, et al. Socioeconomic, demographic and lifestyle factors associated with dietary patterns of women living in Southern Brazil. Cad Saúde Pública. 2009;25(6):1297-306.

4. Ministério da Saúde (BR), Secretaria de Atenção à Saúde, Departamento de Atenção Básica. Guia alimentar para a população brasileira. $2^{\mathrm{a}}$ ed. Brasília: Ministério da Saúde; 2014.

5. Souza SMFC, Lima KC, Miranda HF, Cavalcanti FID. Utilização da informação nutricional de rótulos por consumidores de Natal, Brasil. Rev Panam Salud Pública. 2011;29(5):337-43.

6. Nestle M, Ludwig DS. Front-of-package food labels: public health or propaganda? JAMA. 2010;303(8): 771-2.

7. Câmara MCC, Marinho CLC, Guilam MC, Braga AMCB. A produção acadêmica sobre a rotulagem de alimentos no Brasil. Rev Panam Salud Pública. 2008;23(4):52-8.

8. Ministério da Saúde (BR), Agência Nacional de Vigilância Sanitária. Resolução da Diretoria Colegiada - RDC no 54: Regulamento Técnico Mercosul Sobre Informação Nutricional Complementar. Brasília: Ministério da Saúde; 2012.

9. Ministério da Saúde (BR), Agência Nacional de Vigilância Sanitária. Resolução da Diretoria Colegiada - RDC no 359: Rotulagem geral de alimentos embalados. Brasília: Ministério da Saúde; 2003.

10. Ministério da Saúde (BR), Agência Nacional de Vigilância Sanitária. Resolução da Diretoria Colegiada - RDC no 360: Informação nutricional. Brasília: Ministério da Saúde; 2003.

11. Ferreira $A B$, Lanfer-Marquez UM. Legislação brasileira referente à rotulagem nutricional de alimentos. Rev Nutr. 2007;20(1):83-93.
12. Lobanco CM, Vedovato GM, Cano CB, Bastos DHM. Fidedignidade de rótulos de alimentos comercializados no município de São Paulo, SP. Rev Saúde Pública. 2009;43(3):499-505.

13. Brownell KD, Kersh R, Ludwig DS, Post RC, Puhl RM, Schwartz MB, et al. Personal responsibility and obesity: A constructive approach to a controversial issue. Health Aff. 2010;29(3):379-87.

14. Monteiro RA, Coutinho JG, Recine E. Consulta aos rótulos de alimentos e bebidas por freqüentadores de supermercados em Brasília, Brasil. Rev Panam Salud Publica. 2005;18(3):172-7.

15. Marins BR, Jacob SDC, Peres F. Avaliação qualitativa do hábito de leitura e entendimento: recepção das informações de produtos alimentícios. Ciênc Tecnol Aliment. 2008;28(3):579-85.

16. Basit A, Riaz M, Fawwad A. Improving diabetes care in developing countries: The example of Pakistan. Diabetes Res Clin Pr. 2015;107(2):224-32

17. Haas L, Maryniuk M, Beck J, Cox CE, Duker P, Edwards L, et al. National standards for diabetes selfmanagement education and support. Diabetes Care. 2013; 36 Suppl 1(January):S100-S108.

18. Cunha M, André S, Granado J, Albuquerque C, Madureira A. Empowerment and adherence to the therapeutic regimen in people with diabetes. Procedia Soc Behav Sci. 2015;171:289-93.

19. Chen L, Pei J-H, Kuang J, Chen H-M, Chen Z, Li $\mathrm{Z}-\mathrm{W}$, et al. Effect of lifestyle intervention in patients with type 2 diabetes: a meta-analysis. Metabolism. 2015;64(2):338-47.

20. Lara BR, Paiva VSF. A dimensão psicossocial na promoção de praticas alimentares saudáveis. Interface (Botucatu). 2012;16(43):1039-54.

21. Ministério da Saúde (BR), Secretaria de Atenção à Saúde, Departamento de Atenção Básica. Diretrizes para o cuidade das pessoas com doenças crônicas nas redes de atenção à saúde e nas linhas de cuidado prioritárias. $1^{\text {a }}$ ed. Brasília: Ministério da Saúde; 2013.

22. Evert AB, Boucher JL, Cypress M, Dunbar SA, Franz MJ, Mayer-Davis EJ, et al. Nutrition therapy recommendations for the management of adults with diabetes. Diabetes Care. 2014;37(Suppl 1):120-43.

23. Araújo WM, Montebello NP, Botelho RBA, Borgo LA. Alquimia dos alimentos. $2^{\mathrm{a}}$ ed. Brasília: Editora Senac; 2008. 
24. Barreiros RC. Adoçantes nutritivos e não-nutritivos. Rev Fac Ciênc Méd Sorocaba. 2012;14(1):5-7.

25. Jardim ANO, Caldas ED. Exposição humana a substâncias químicas potencialmente tóxicas na dieta e os riscos para a saúde. Quim Nov. 2009;32(7):1898909.

26. Ministério da Saúde (BR), Agência Nacional de Vigilância Sanitária. Resolução da Diretoria Colegiada - RDC no 40: Regulamento Técnico para Rotulagem Nutricional Obrigatória de Alimentos e Bebidas Embalados. Brasília: Ministério da Saúde; 2001.

27. Goh Y-Y, Bogart LM, Sipple-Asher BK, Uyeda K, Hawes-Dawson J, Olarita-Dhungana J, et al. Using community-based participatory research to identify potential interventions to overcome barriers to adolescents' healthy eating and physical activity. J Behav Med. 2009;32(5):491-502.
28. Oakley P, Clayton A. Monitoramento e avaliação do empoderamento [Internet]. São Paulo: Instituto Pólis; 2003 [acesso em 20/07/2014]. Disponível em: http:// www.polis.org.br/uploads/928/928.pdf

29. Magalhães P, Motta DG. Uma abordagem psicossocial do estado nutricional e do comportamento alimentar de estudantes de nutrição. Nutrire Rev Soc Bras Aliment Nutr. 2012;37(2):118-32.

\section{Endereço para correspondência:}

Anna Cecília Queiroz de Medeiros

Faculdade de Ciências da Saúde do Trairi

Universidade Federal do Rio Grande do Norte

Rua Vila Trairi, S/N

Bairro: Centro

CEP: 59200-000 - Santa Cruz - RN - Brasil

E-mail: annacqm@yahoo.com.br 